\title{
HOMOMORPHISM AND SIGMA POLYNOMIALS
}

\author{
RICHARD ALAN GILLMAN \\ Department of Mathematics and Computer science \\ valparaiso University, Valparaiso IN, 46383
}

(Received October 4, 1993 and in revised form February 16, 1995)

ABSTRACT. By establishing a connection between the sigma polynomial and the homomorphism polynomial, many of the proofs for computing the sigma polynmial are simplified, the homomorphism polynomial can be identified for several new classes of graphs, and progress can be made on identifying homomorphism polynomials.

KEY WORDS AND PHRASES. graph homomorphisms, graph polynomials 1992 AMS SUBJECT CLASSIFICATION CODE. 05C15

1. INTRODUCTION.

Throughout this paper, a graph will refer to a simple graph, and for a given graph $G$, let $G_{L}$ be the graph obtained by inserting an edge between two non-adjacent vertices, let $G_{R}$ be the graph obtained by obtained by contracting the edge inserted in $G_{L}$. Thus $G=G_{L}+G_{R}$ is the standard decomposition of $G$ used for obtaining the chromatic polynomial of $G$. Further, let $G_{p}$ be the insertion of a pseudo-edge between two non-adjacent vertices (pictorially, a pseudo-edge is a dashed edge between the two vertices: we will interpet this to mean that the vertices are still non-adjacent, but we have considered this pair of vertices already), in which case $G_{R}$ denotes the graph obtained by contracting the pseudo-edge. A graph is pseudo-complete if every pair of vertices is either adjacent or pseudo-adjacent. We denote a pseudo-complete graph by $K_{p(m)}$, where $p$ is the number of vertices and $m$ is the number of pseudo-edges.

A homomorphism from $G$ onto a graph $G^{\prime}$ is a mapping $\phi$ from $V(G)$ onto $V\left(G^{\prime}\right)$ such that if vertices $u$ and " aro adjaront in $G$, then $\phi(u)$ and $\phi(v)$ are adjacent in $G^{\prime}$. Bari, [1], showed that the set of all homomorphic images of $G, h(G)$, can be found by using the recursion

$$
h(G)=h\left(G_{P}\right) \cup h\left(G_{R}\right) \text {, }
$$


which terminates when each graph is pseudo-complete. We find the set of homomorphic images of $G$ by ignoring the pseudo-edges in these graphs.

For a $\left(p,\left(\frac{p}{2}\right)-m\right)$ graph, this recursive process yields the homomorphism polynomial

$$
h(G ; x, y)=\sum_{p(a)} \sum_{H} x^{p} y^{m},
$$

where $H$ is the set of pseudo-complete graphs obtained in the final step of the recursive process. As discussed by Bari, [1], the homomorphism polynomial has several inter sting properties, including the fact that it yields the chromatic number $\chi(G)$ as the minimum degree of the terms of $h(G ; x, 0)$ and that the chromatic polynomial of $G, P(G ; \lambda)$, can be obtained from $h(G ; x, 1)$ by replacing the $x^{n}$ with the falling factorial $\lambda^{(n)}=\lambda(\lambda-1) \ldots(\lambda-n+1)$. For example,

$$
h\left(c_{4} ; x, y\right)=x^{4} y^{2}+2 x^{3} y+x^{2}
$$

and

$$
P\left(C_{4} ; \lambda\right)=\lambda^{(4)}+2 \lambda^{(3)}+\lambda^{(2)} \text {. }
$$

Korfhage, [5], introduced the sigma polynomial of $G$ and defined it as follows. For all $\mathrm{p}, \sigma\left(\mathrm{K}_{\mathrm{p}} ; s\right)=1$. If $\mathrm{G}$ is not complete, suppose that $\sigma\left(G_{L} ; s\right)$ is a polynomial of degree $m$ and $\sigma\left(G_{R} ; s\right)$ is a polynomial of degree $n$, then $\sigma(G ; s)$ is a polynomial of degree $\max \{n, m+1\}$, defined by

$$
\begin{array}{ll}
\sigma(G)=\sigma\left(G_{L} ; s\right) \cdot s^{n-m+1}+\sigma\left(G_{R} ; s\right), & \text { if } n \geq m+1 \\
\sigma(G)=\sigma\left(G_{L} ; s\right)+\sigma\left(G_{R} ; s\right) \cdot s^{m-n+1}, & \text { if } n<m+1 .
\end{array}
$$

It was introduced as a way to investigate the chromatic polynomial, and can be obtained from the $P(G, \lambda)$ in the following manner. If we write the chromatic polynomial of the $(p, q)$-graph $G$ in falling factorial form,

$$
P(G ; \lambda)=\sum_{i=0}^{p} a_{i} \lambda^{(p-i)}
$$

then

$$
\sigma(G ; s)=\sum_{i=0}^{k} a_{i} s^{k-i},
$$

where $k=p-\chi(G)$. Thus, as an example, we have

$$
P\left(C_{4} ; \lambda\right)=\lambda^{(4)}+2 \lambda^{(3)}+\lambda^{(2)}
$$

and

$$
\sigma\left(c_{4} ; s\right)=s^{2}+2 s+1
$$

As our examples illustrate, there is a connection between the homomorphism polynomial and the sigma polynomial. By utilizing their relationship to the chromatic polynomial we obtain the following theorem.

THEOREM 1. For any graph $G, h(G ; s, 1) \cdot s^{-x}=\sigma(G ; s)$. 
PROOF. Since $P(G ; \lambda)=h(G ; x, 1)$ with $x^{n}$ replaced with $\lambda^{(n)}$, and $\sigma(G ; s)=P(G ; \lambda) / \lambda(x)$ with $\lambda(n)$ replaced with $s^{n}$, the result follows by direct substitution of $s$ for $x$ in $h(G ; x, 1) x^{-\chi}$.

By utilizing this theorem, we can obtain many of the computational formulas for $\sigma(G ; s)$ from those given for $h(G ; x, y)$. We will do this in section 2. Further, the work done on characterizing graphs with certain sigma polynomials will further the classification process for homomorphism polynomials. We will investigate these processes in sections 3 and 4.

2. REDUCTION THEOREMS.

A wide variety of reduction and characterization theorems concerning homomorphisms appear in Girse, [4]. We provide two examples here to illustrate their range, and their relationship to results on the sigma polynomial. The corollaries given here are found originally in Korfhage's work, [5], but follow directly from the theorems by applying Theorem 1.

THEOREM 2. If $G=G_{1}+G_{2}$ (the join), then

$$
h(G ; x, y)=h\left(G_{1} ; x, y\right) \cdot h\left(G_{2} ; x, y\right) \text {. }
$$

COROLLARY 2.1. For any graph $G, \sigma(G+v ; s)=\sigma(G ; s)$, where $G+v$ is the join of the vertex $v$ and $G$.

THEOREM 3. If $G=k_{p_{1}} \cup k_{p_{2}}$, where $p_{1} \geq p_{2}$, then

$$
h(G ; x, y)=\sum_{k=0}^{p_{2}} k !\left(\begin{array}{l}
p_{1} \\
k
\end{array}\right)\left(\begin{array}{l}
p_{2} \\
k
\end{array}\right) x p_{1}+p_{2}-k y^{\left(p_{1}-k\right)\left(p_{2}-k\right)} \text {. }
$$

COROLLARY 3.1. $\sigma\left(K_{p_{1}} \cup K_{p_{2}} ; s\right)=\sum_{i=0}^{p_{2}}\left(\begin{array}{l}p_{i} \\ i^{2}\end{array}\right) p_{1}\left(p_{2}-i\right) s^{i}, p_{2} \leq p_{1}$.

However, not all of the characterization theorems concerning sigma polynomials are derivable from corresponding results on homomorphism polynomials. For example, if we let $\mathrm{H}$ be a graph with distinct vertices $u$ and $v$, let $w$ be a vertex not in $H$, let $G_{1}$ be $H u w$ with the edge $u w$, and let $G_{2}$ be $H \cup v$ with the edge $v w$, then we have the following theorem and corollary from Korfhage, [5].

THEOREM 4. $\sigma\left(G_{1} ; s\right)=\sigma\left(G_{2} ; s\right)$.

COROLLARY 4.1. All trees on $p+1$ vertices have the same sigma polynomial.

More general versions of these do not hold concerning homomorphism polynomials; specifically, empirical evidence suggests that every tree has a distinct homomorphism polynomial.

3. SPECIFIC CLASSES.

The sigma polynomial for several classes of graphs have been identified, and the techniques used to do this can be generalized to identify the homomorphism polynomials of these classes. Our first results in this direction are modifications of similar results in Korfhage, [5]. 
THEOREM 5. Let $G_{k}$ be a $(2 k-2)$ regular graph on $p=2 k$ vertices. Then

$$
h\left(G_{k} ; x, y\right)=\left(x+x^{2} y\right) h\left(G_{k-1} ; x, y\right)=\sum_{i=x}^{p}\left(\begin{array}{c}
p \\
i-x
\end{array}\right) x^{i} y^{i-x} .
$$

PROOF. We have

$$
h\left(G_{k} ; x, y\right)=h\left(G_{k_{p}} ; x, y\right)+h\left(G_{k_{R}} ; x, y\right) .
$$

But $G_{k_{R}}$ is a vertex joined to $G_{k-1}$ and $G_{k_{P}}$ is $\bar{k}_{2}$ joined to $G_{k-1}$, so that

$$
h\left(G_{k_{R}} ; x, y\right)=(x) h\left(G_{k-1} ; x, y\right)
$$

and

$$
h\left(G_{k_{P}} ; x, y\right)=\left(x^{2} y\right) h\left(G_{k-1} ; x, y\right)
$$

Thus, the recursion follows, and the polynomial follows from the fact that $h\left(G_{2} ; x, y\right)=x^{2} y+x$.

THEOREM 6 . Let $G$ be the graph on $n+2$ vertices, with $n+1$ vertices of degree $n$ and one vertex of degree 2 . If $n>1$,

$$
h(G ; x, y)=x^{n+2} y^{n}+\left((n-1) y+y^{n-1}\right) x^{n+1}+(n-1) x^{n} .
$$

PROOF. Let $v$ be the vertex of degree 2 , with neighbors $u$ and $w$. since $u, w$, and all other vertices not adjacent to $v$ are of degree $n$, $u$ and $w$ must not be adjacent. Thus there are two types of elecentary homomorphisms possible: identifying $u$ and $w$, or identifying $v$ to another vertex. Thus the result follows from direct computation.

4. QUADRATIC FORMS.

In this section, we will use the fact that if $h(x, y)$ is the homomorphism polynomial of some graph, then $h(s, 1) s^{-\chi}$ is the sigma polynomial of the same graph, and the fact that a graph $G$ has a sigma polynomial of the form

$$
s^{2}+m s+c
$$

if and only if the homomorphism polynomial of $G$ is of the form

$$
x^{p_{y}}+P(y) x^{p-1}+c x^{p-2} \text {, }
$$

where $P(Y)$ is a polynomial in $y$ with coefficients summing to $m$. The latter follows from the fact that $G$ has a quadratic sigma polynomial if and only if $G$ has chromatic number $p-2$, which is true if and only if the smallest term of the homomorphism polynomial of $G$ is of the form $C x^{p-2}$.

We obtain the following theorems by generalizing similar results concerning sigma polynomials.

From Theorem 5 in $X u,[7]$, we can get the following bounds on the coefficients of $h(G ; x, y)$. 
THEOREM 7. Let $G$ be a $(p, q)$ graph with homomorphism polynomial

Then, for $1=x_{1} \ldots, p$,

$$
h(G ; x, y)=\sum_{i=x}^{p} P_{i}(y) x^{i} \text { and let } m=\left(\begin{array}{l}
p \\
2
\end{array}\right)-q \text {. }
$$

$$
P_{i}(1) \leq \min \left\{\left(\begin{array}{c}
m \\
i+m-p
\end{array}\right),\left\{\begin{array}{c}
p \\
p-i
\end{array}\right\}\right\} .
$$

where $\left\{\begin{array}{c}p \\ p-i\end{array}\right\}$ is a stirling number of the second kind.

We introduce the following graphs, first defined by Frucht and Giudici in [3], to obtain a characterization of certain homomorphism polynomials. The graph $T(a, b, c, d)$ has two distinquished vertices $t_{1}$ and $t_{2}$, which are adjacent to each other. There are a vertices adjacent to both $t_{1}$ and $t_{2} ; b$ vertices adjacent to $t_{1}$ only; $c$ vertices adjacent to $t_{2}$ only; and $d$ isolated vertices. Without loss of generality, we can let $b \geq c$, and to avoid trivial graphs, assume $a+c>0$. The graph $U(a, b, c, d)$ is obtained from $T(a, b, c, d)$ by deleting the edge between $t_{1}$ and $t_{2}$.

From work by Frucht and Giudici, [3], and Li and whitehead, [6], concerning these graphs and quadratic sigma polynomials, we obtain the following theorem, with the specific polynomials found by direct computation.

THEOREM 8. A graph $G$ has a homomorphism polynomial of the form $x^{p} y^{m}+P(y) x^{p-1}+c x^{p-2}$.

where $P(1)=m$ and $C$ is constant, if and only if the complement of $G$ is either $c_{5} \cup d K_{1}, T(a, b, c, d)$, or $U(a, b, c, d)$ different from

$U(1,0,0, d)$ where $a+c>0$. In fact,

$$
\begin{aligned}
& h\left(\overline{c_{5} \cup d K_{1}}\right)=x^{d+5} y^{5}+5 x^{d+4} y^{2}+5 x^{d+3} \\
& h(\overline{T(a, b, c, d)})=x^{p+2} y^{2 a+b+c+1} \\
& +x^{p+1}\left(y^{a}+b y^{a+c}+a y^{a+c}+(a+c) y^{a+b}\right) \\
& +x^{p}(a+b)(a+c)
\end{aligned}
$$

and

$$
\begin{aligned}
& h(\overline{U(a, b, c, d)})=x^{p+2} y^{2 a+b+c}+x^{p+1}\left(b y^{a+c}+a y^{c}+a y^{b}+c y^{a+b}\right) \\
& +x^{p}(b(a+c)+a(a+c-1))
\end{aligned}
$$

other characterizations of quadratic sigma polynomials found by Dhurandhur, [2], Korfhace. [5], and $x_{11},[7]$, can be generalized for homomorphism polynomials. For example, we can obtain the following theorem.

THEOREM 9. The polynomial $x^{p} y^{m}+p(y) x^{p-1}+c x^{p-2}$, where $P(1)=m$, is a homomorphism polynomial for some graph $G$ iff 
$m-2 \leq c \leq m^{2} / 4$, and there exists an integer $b \leq m / 2$ such tnat $b(m-1-b) \leq c \leq b(m-b)$.

Results similar to these can be generated for homomorphism polynomials of the form

$$
x^{p_{y} m}+P_{p-1}(y) x^{p-1}+p_{p-2}(y) x^{p-2}+C x^{p-3}
$$

by generalizing the related work by Dhurandhur, [2].

\section{REFERENCES}

[1] BARI R., Homomorphism polynomials of graphs, Journal of Combinatorics, Information $\underline{\&}$ Systems Sciences, v7 $\$ 1$ p56-64 (1982).

[2] DHURANDHUR M., Characterization of quadratic and cubic $\sigma$-polynomials, Journal of Combinatorial Theory Series B, 137 p210-220 (1984).

[3] FRUCHT R. and GIUDICI R., Some chromatically unique graphs with seven points, Ars Combinatorica, v16A p161-172 (1983).

(4) GIRSE R., Homomorphism polynomials of certain graphs, Journal of combinatorics, Information $\underline{\&}$ Systems Sciences, v10 p62-68 (1985).

[5] KORFHAGE R., $\sigma$-polynomials and graph coloring, Journal of Combinatorial Theory Series B, v24 p137-153 (1977).

[6] LI N. and WHITEHEAD E., Characterizations of graphs having quadratic $\sigma$-polynomials, unpublished.

[7] XU S., On $\sigma$-polynomials, Discrete Mathematics, v69 p189-194 (1988). 


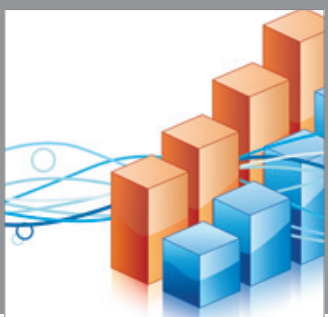

Advances in

Operations Research

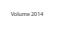

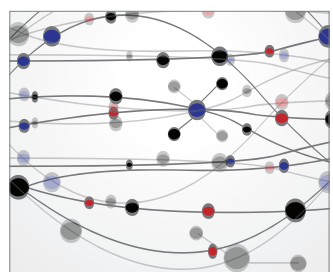

\section{The Scientific} World Journal
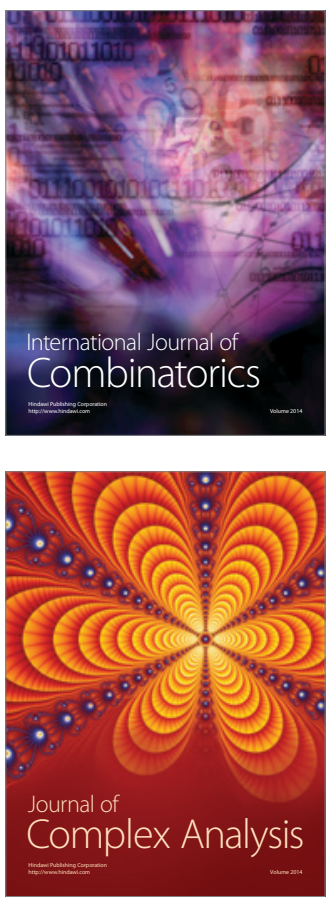

International Journal of

Mathematics and

Mathematical

Sciences
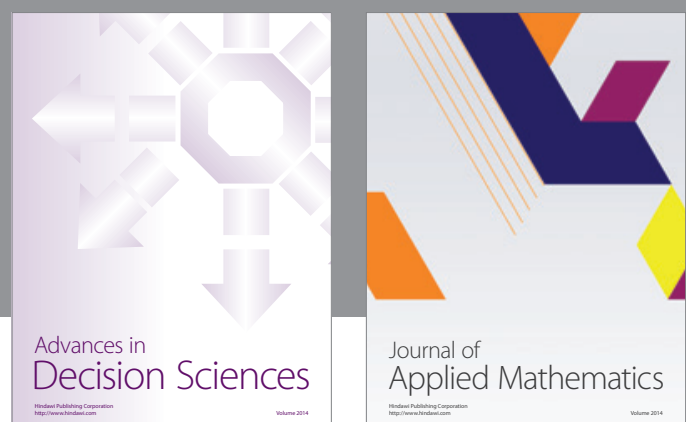

Journal of

Applied Mathematics
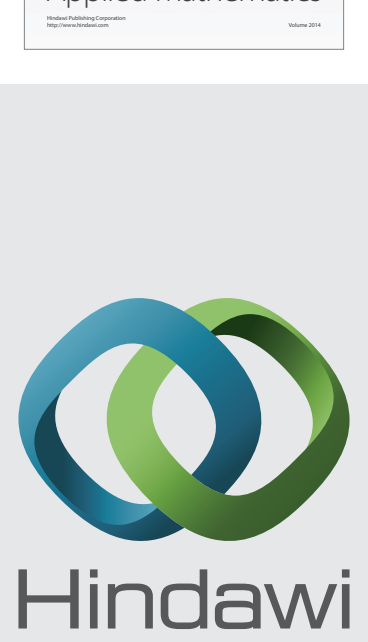

Submit your manuscripts at http://www.hindawi.com
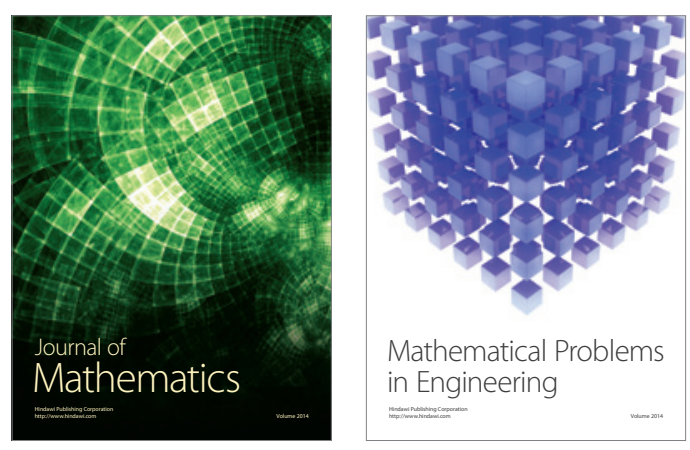

Mathematical Problems in Engineering
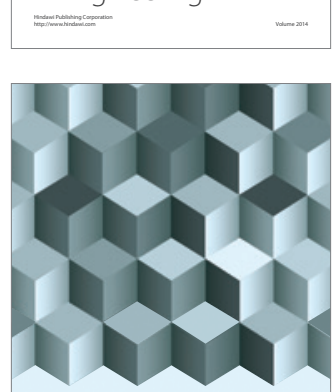

Journal of

Function Spaces
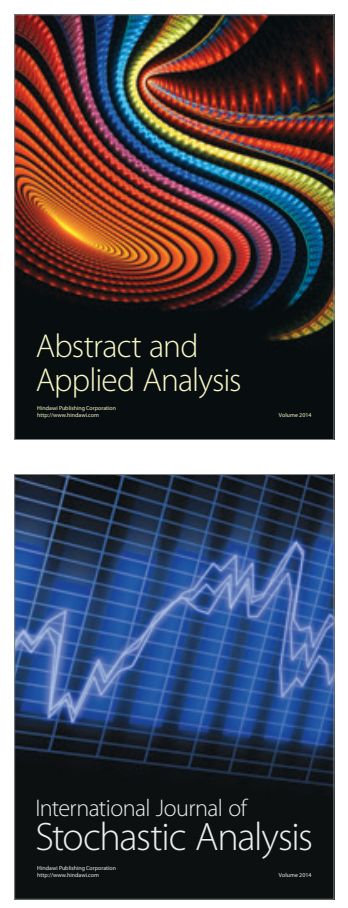

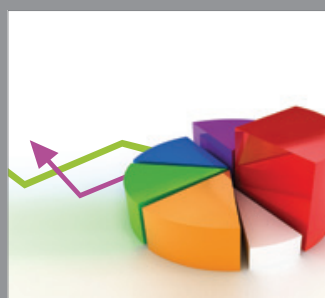

ournal of

Probability and Statistics

Promensencen
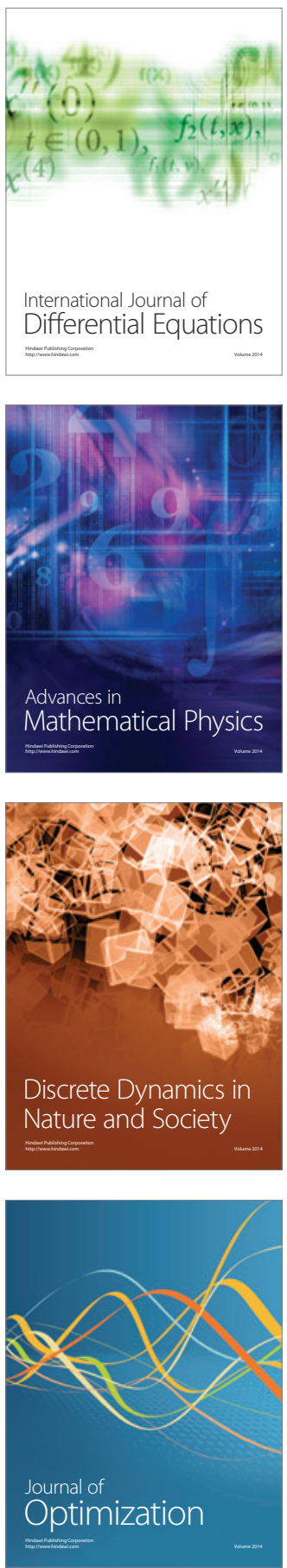